\title{
Delay Compensation for Real Time Disturbance Estimation at Extremely Large Telescopes
}

\author{
Michael Böhm, Jörg-Uwe Pott, Martin Kürster, Oliver Sawodny, Denis Defrère, Phil Hinz
}

\begin{abstract}
In ground-based astronomy, aberrations due to structural vibrations, such as piston, limit the achievable resolution and cannot be corrected using adaptive optics for large telescopes. We present a model-free strategy to estimate and compensate piston aberrations due to vibrations of optical components using accelerometer disturbance feedforward, eventually allowing the use of fainter guide stars both for the fringe detector and in the adaptive optics loop (AO-loop). Because the correction performance is very sensitive to signal delays, we present a strategy to add a delay compensation to the developed disturbance estimator, which can in principle be applied to many other applications outside of astronomy that lack observer performance due to a measurement delay or need a prediction to compensate for input delays. The ability to estimate vibration disturbances in the critical frequency range of $8 \mathrm{~Hz}$ to $60 \mathrm{~Hz}$ is demonstrated with on sky data from the Large Binocular Telescope Interferometer (LBTI), an interferometer at the Large Bincoular Telescope (LBT). The experimental results are promising, indicating the ability to suppress differential piston induced by telescope vibrations by a factor of about 3 (RMS), which is significantly better than any currently commissioned system.
\end{abstract}

Index Terms-acceleration, disturbance, delay, optics, sensor networks, estimation, prediction, telescope

\section{INTRODUCTION}

$\mathbf{L}$ ARGE ground-based Telescopes have undergone tremendous growth in size in the past decades. Today, the largest single monolithic mirrors for telescopes have diameters of approximately $8 \mathrm{~m}$, which constitutes a manufacturing limit. Thus, some past and most future telescope projects going beyond the $8 \mathrm{~m}$-class rather utilize an array of single mirrors to increase the effective resolution [1], [2], [3]. One of these is the Large Binocular Telescope (LBT) located on Mt. Graham near Tucson, Arizona (USA), which is equipped with two large $8.4 \mathrm{~m}$ primary mirrors, as shown in Figure 1 . The light collected by both primary mirrors is reflected via the adaptive secondary mirrors and tertiary mirrors into the middle of the telescope, where different astronomic instruments reside. An astronomic instrument contains optics, optical sensors and scientific cameras used to analyze the incoming telescope light and aquire the astronomical image of the sky. The tertiary mirrors can be turned to move the focus to any of the instruments in the middle of the telescope. For the LBT, two of these center-positioned instruments are interferometers - the Large Binocular Telescope Interferometer (LBTI) built by the University of Tucson and described in [4], and the

M. Böhm and O. Sawodny are with the Institute for System Dynamics, University of Stuttgart, Germany, email: (see contact info at www.isys.unistuttgart.de)

J.-U. Pott and M. Kürster are with the Max-Planck-Insitute for Astronomy.

D. Defrère and P. Hinz are with the University of Arizona

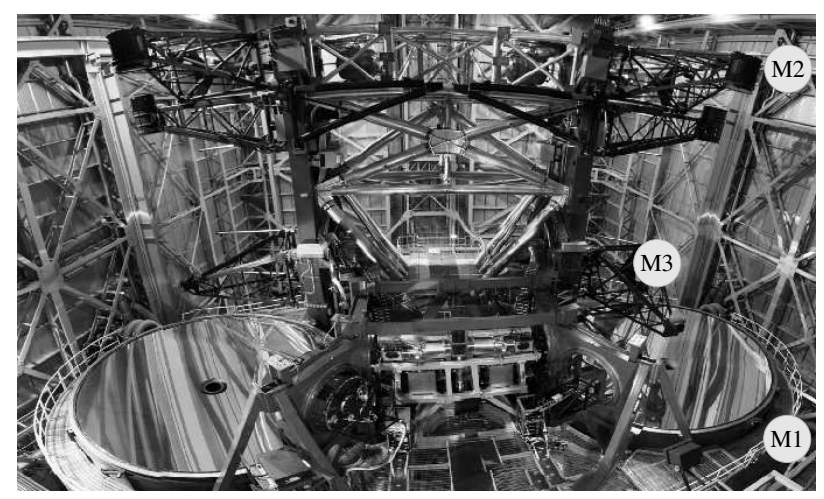

Fig. 1. Inner part of the Large Binocular Telescope with its symmetrical layout. The optical setup can be changed and is set according to the observing instrument.

LBT INterferometric Camera for Near-InfraRed and Visible Adaptive INterferometry for Astronomy (LINC-NIRVANA), which is a stellar interferometer built by a German consortium led by the Max-Planck-Institute for Astronomy in Heidelberg, Germany and described in [5]. For interferometry, the spatial resolution is determined by the largest baseline, which means the maximal distance of any two points on the primary mirrors. For the LBT, this baseline is $22.8 \mathrm{~m}$ for LINC-NIRVANA and $15 \mathrm{~m}$ for LBTI. So on one hand, it is not necessary to increase the size of the single optical components in order to increase the optical performance, on the other hand, due to several sources of disturbance excitations, need arises for an opto-mechanical device to correct the difference in the optical path (optical pathway difference - OPD) between both sides to keep both light paths co-phased, which is necessary for interferometric imaging [6]. While the most important disturbance source is wind excitation [7], significant other contributors can be cooling systems and/or electrical drives [8], for example. For LINC-NIRVANA, this device is a positioncontrolled piezo drive with a travel range of $75 \mu \mathrm{m}$. The internal controller was developed at an earlier stage of the project [9]. On the contrary, LBTI uses a kind of tweeterwoofer approach, with one slower piezo drive having a larger travel range to compensate for slow but large differences and one smaller, faster one with a small travel range for disturbances of higher frequencies [4]. We will focus on LBTI, for which measurement results will be presented. However, a good delay compensation is even more important for LINCNIRVANA, due to its larger input delay to the compensation device, the cause of which will be explained in more detail at the end of Section IV. 


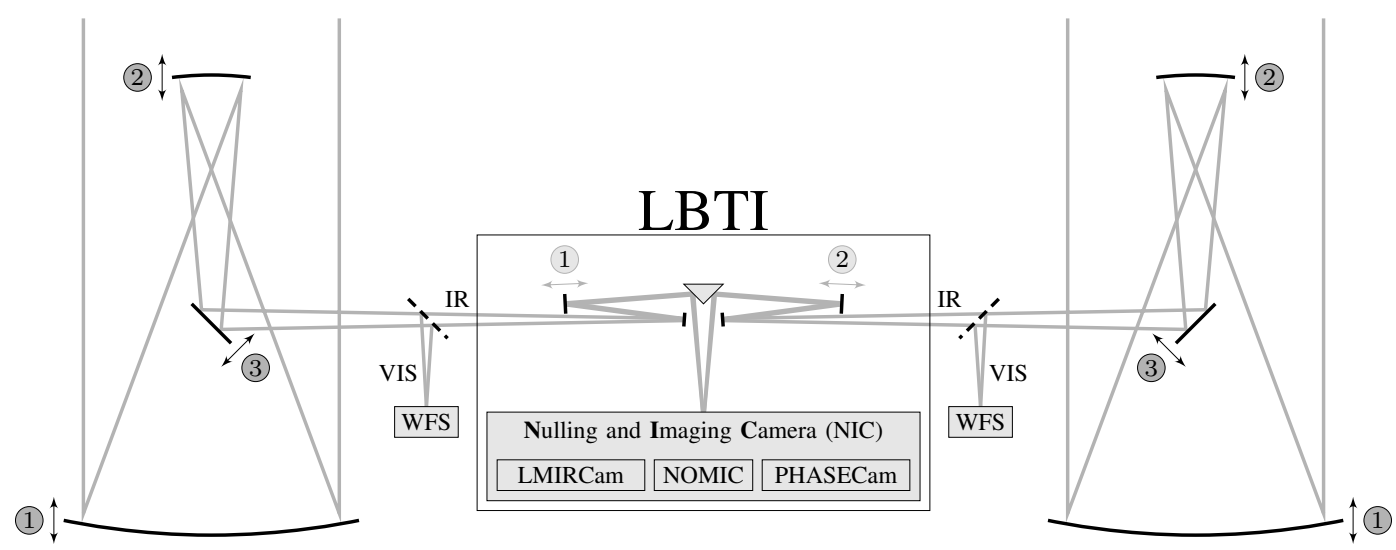

Fig. 2. Optical layout of the LBT with LBTI (only infrared light path, according to [4]), including the tweeter-woofer setup composed of a fast (1) and a slow (2) piston corrector. The mirror motion of M1, M2 and M3 (1) - (3) on both sides due to windloads is measured using 3 accelerometers along the optical axis of each mirror. The two wavefront sensors use visible light, while the near-infrared (NIR) light is collected in the cameras of LBTI.

In this paper, we are concerned with using acceleration measurements at each mirror to estimate the mirror's displacement along the optical axis and thus calculate the OPD between both sides of the telescope in order to use these values as inputs to the correction devices. So far, each mirror is equipped with five accelerometers. In the current configuration of the optical vibration measurement system (OVMS), as described in [10], three accelerometers measure accelerations normal to the mirror surface (called the $z$-axis), which can be used to calculate the vertical displacement of the mirror and two rotational degrees of freedom, which in optics are referred to as piston, tip and tilt mode, respectively. Those are the three degrees of freedom for out-of-plan motion of the mirror, where each mirror's contribution to the OPD is directly proportional to its displacement, or piston. This is the specific LBT setup, but the described algorithm can be generally applied to estimate and compensate disturbances leading to piston (OPD), tip and tilt aberration along the optical path using acceleration measurements at Large Telescopes, as illustrated by [11] for the E-ELT. In theory, OPD variations resulting from mirror oscillations could also be corrected by pure feedback using image processing algorithms along with the respective fringe tracking detector. This detector is located in the interferometric instrument and uses nearinfrared interference fringes to measure the OPD between the two beams. For a scheme of the optical layout of the LBT and the instrument LBTI, refer to Figure 2. However, a fast fringe tracking detector readout would be required, which means short integration times and flux limitations for faint stars. Thus, as described in [12] in more detail, the fast correction for the higher OPD-frequencies is done by a seperate system using accelerometers to measure the OPD-disturbances and a piezo driven opto-mechanical correction device( the piston mirror for LINC-NIRVANA and the Fast and Slow Pathlength Correctors (FPC and SPC) for LBTI) to correct for them, while the detector can be used in a relatively slow fashion. This idea of a disturbance feedforward is summarized in Figure 4. The disturbance $d(t)$ causes an OPD $o_{\mathrm{T}}$ between the left and right telescope side, which is observed by measuring the accelerations $\boldsymbol{y}=\left[y_{1}, y_{2}, \ldots, y_{n}\right]^{\top}$ with accelerometers installed at each one of the telescope mirrors (currently five sensors per mirror). The estimated OPD $\hat{o}_{\mathrm{T}}$ is then calculated from the estimated sensor motions $\boldsymbol{x}(t)=\left[x_{1}, x_{2}, \ldots, x_{n}\right]^{\top}$ according to the telescope geometry and the sensor positions and fed to the respective correction device for compensation. Additionally, feedback can be applied using image detection algorithms with a much smaller sampling rate. Using this so called Fringe and Flexure Tracking System to estimate the remaining OPD after disturbance feedforward, slow frequency oscillations, e.g. due to telescope tracking motion, can also be reduced very efficiently.

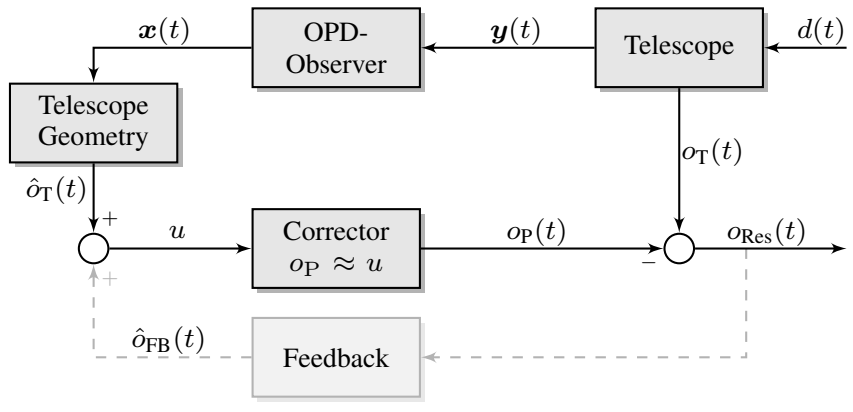

Fig. 4. Disturbance compensation scheme

The telescope's mirrors show very different vibration characteristics, as can be seen in Figure 3. Whereas M1 is very heavy (about $8 \mathrm{t}$ ) and thus does not tend to vibrate more than a few $100 \mathrm{~nm}$, the very leightweight support structure of M2 and M3 leads to very low eigenfrequencies between $11 \mathrm{~Hz}$ and $14 \mathrm{~Hz}$ for M2 and starting at $18 \mathrm{~Hz}$ for M3. Thus, these mirrors tend to vibrate strongly with significant amplitudes of several $\mu \mathrm{m}$, even under "normal" weather conditions. The goal is to keep the OPD smaller then $0.1 \lambda$, with $\lambda$ being the observing wavelength. This is around $100 \mathrm{~nm}$ for the LINC-NIRVANA instrument, working in the near-infrared $(\lambda \in[1,2.5] \mu \mathrm{m})$, whereas LBTI observes at wavelengths above $\lambda=2.5 \mu \mathrm{m}$, relaxing the requirement on OPD compensation slightly.

Additionally, due to network communication delays and 


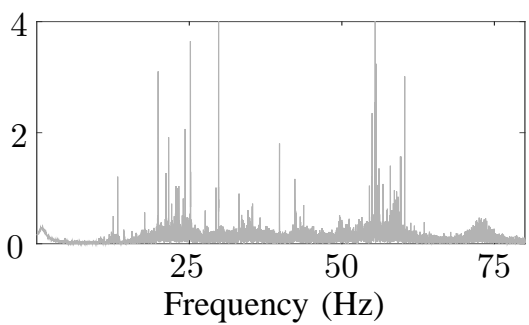

(a) primary mirror (M1)

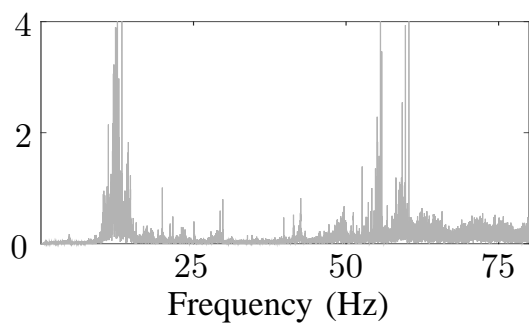

(b) secondary mirror (M2)

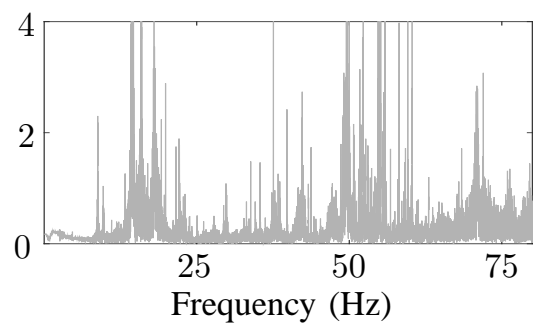

(c) tertiary mirror (M3)

Fig. 3. Example acceleration amplitude spectrum for an observing night, where open loop fringes could be aquired by LBTI due to slow wind speeds (Feb. 4, 2015, 08:34 UTC). Amplitudes are given in $\frac{\mathrm{mm}}{\mathrm{s}^{2}}$. The data shows an average over the three sensors along the optical axis at each of the main mirrors on the right side. Sensor positions are according to [10]

actuator input delays, a delay compensation is necessary to reduce the OPD to a tolerable level. For adaptive optics systems, delay compensation has been studied extensively, for example as early as 1993 in [13]. Most of these methods employ some kind of model based prediction based on an atmospheric model, e.g. in [14]. However, for structural vibrations, this approach would completely fail, since these vibrations cannot be predicted by this model. The presented delay compensation can be used for any observers used in feedback or feedforward structures, and one would have to study the coupling between several correction loops such as $\mathrm{AO}$ and fringe tracking. However, at the LBT, the delay compensating observer is only used for the feedforward OPD compensation, and there is thus no coupling with the AO control loop.

Thus, two main goals to accomplish the task of disturbance compensation for the LBT can be identified. First, a precise estimation of the mirror positions is needed, and second, a delay compensating algorithm needs to introduce the necessary phase to anticipate the time delay. The first problem, the estimation of the mirror positions, is dicussed in section II, while the second, the delay compensation algorithm, is presented in section III. Section IV will present measurement results from the LBT taken by the LBTI-team.

\section{Estimation Algorithm}

\section{A. Problem description}

In order to derive the input for the piston mirror, each mirror's displacement has to be derived from the accelerometer signals. For simplicity, we will look at a single sensor displacement now without loss of generality, since the derived algorithms can be applied to all sensors and the differential piston can be calculated using sensor placement and telescope geometry information. In general, the sensor displacement $s(t)$ is derived from the real acceleration $a(t)$ at the acceleration sensor by integrating twice:

$$
s(t)=\int_{0}^{t} \int_{0}^{\tau} a(\sigma) \mathrm{d} \sigma \mathrm{d} \tau .
$$

However, this is not applicable, because, for one, the measured acceleration $y_{i}(t)$ is not equal to the real acceleration, but includes a slow signal drift $\eta_{i}^{\text {drift }}(t)$, measurement noise $\eta_{i}^{\text {noise }}(t)$ and an offset $\eta_{i}^{\text {off }}$ :

$$
y(t)=a(t)+\eta^{\mathrm{drift}}(t)+\eta^{\mathrm{noise}}(t)+\eta^{\mathrm{off}},
$$

and for two, the initial velocity cannot be measured and is thus unknown. Due to these factors, $s(t)$ derived from a simple double integration will always be dominated by slow frequency components as $t \rightarrow \infty$. Therefore, the position has to be estimated using a dynamic system to approximate a double integrator in the desired frequency range and suppress the slow frequency components of the signal. Thus, the estimated position $x(t)$ and acceleration measurement $y(t)$ are dynamically related by the general expression

$$
X(s)=E(s) Y(s),
$$

where $E(s)$ represents the Laplace transform of the dynamic system and shall be called Estimator. In earlier publications, we have proposed a model based estimation technique, which has been described in [15] and [16]. Another approach is not model based, but aims at approximating the double integrator for a broader spectrum. It is derived and compared in [15]. Because our delay compensation will be based on this estimator, we will briefly summarize the main idea again in the following subsection. Other approaches for such disturbance estimators can be found in [17], [18] or [19], for example. For the very special task of estimating telescope mirror vibrations, a very basic model-free broadband filtering approach is also described by [20] for the Very Large Telescope Interferometer (VLTI).

\section{B. Algorithm}

In contrast to the model based disturbance observer, the broadband filtering aims at approximating the double integrating behaviour for a specific frequency range, which ideally contains all the dominant eigenfrequencies. For the investigated telescope, this range spans from about $8 \mathrm{~Hz}$ to $60 \mathrm{~Hz}$. However, very low frequencies should be attenuated, to filter out the accelerometer drifts and offsets. An analog filter design for this task is presented by [21], implementing two low pass filters and three highpass filters using passive electrical components and operational amplifiers. Two lowpass filters are needed for approximating the double integration above the cutoff frequencies. Three highpasses are needed to sufficiently suppress small frequency components, otherwise resulting in large offsets in the estimated position. The cutoff for these highpasses has to be chosen according to the frequencies of sensor drifts and low frequency disturbances due to the 

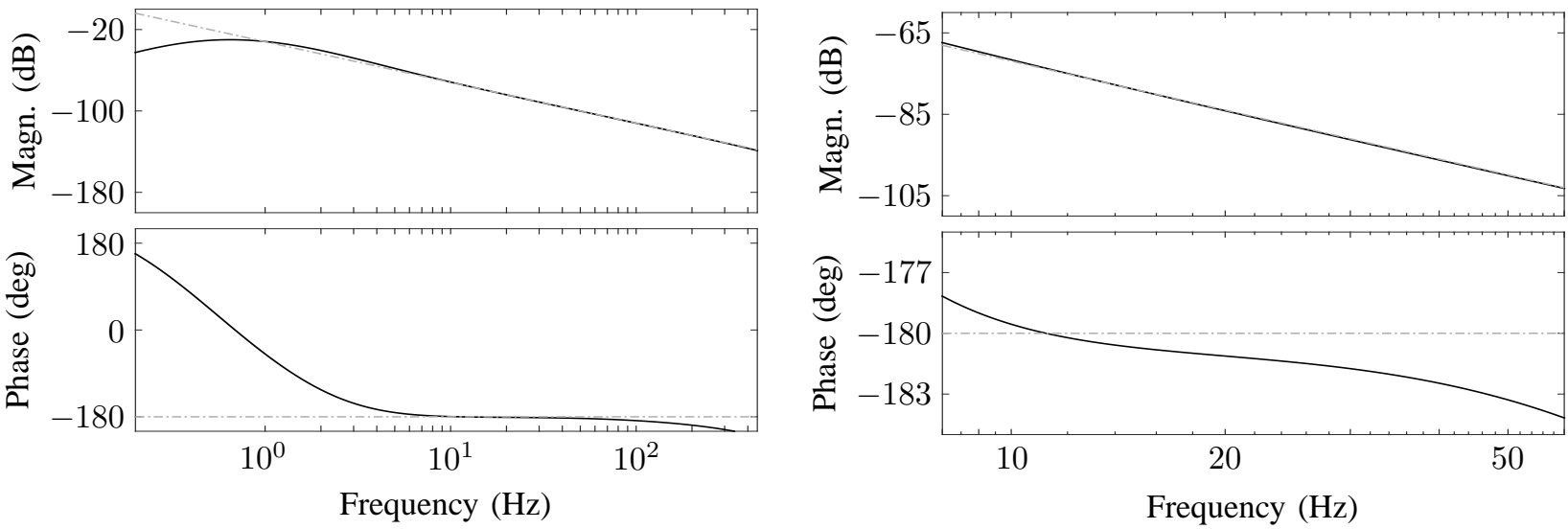

Fig. 5. Bode Diagramm for the broadband filter $G_{F}(s)$, comparing the ideal double integrator (dash-dotted gray) with the designed filter used for position estimation (solid dark) in the complete frequency range (left) and for the desired frequency range of $8 \mathrm{~Hz}$ to $60 \mathrm{~Hz}$ (right).

slow tracking motion of the telescope. In order to sufficiently suppress small frequency components while keeping the phase shift above $8 \mathrm{~Hz}$ at a minimum, we choose a highpass cutoff frequency of $0.5 \mathrm{~Hz}$. Thus, the highpass filter $G_{\mathrm{H}}(s)$ in state space notation is given by the following equation:

$$
\begin{aligned}
& \dot{\hat{\xi}}_{\mathrm{H}}=-\omega_{\mathrm{H}} \hat{\xi}_{\mathrm{H}}+u_{\mathrm{H}}, \\
& y_{\mathrm{H}}=-\omega_{H} \hat{\xi}_{\mathrm{H}}+u_{\mathrm{H}},
\end{aligned}
$$

with $\omega_{\mathrm{H}}=\pi$. The low pass $G_{\mathrm{L}}(s)$ is chosen with a cutoff frequency of $1.5 \mathrm{~Hz}$ and given by

$$
\begin{aligned}
& \dot{\hat{\xi}}_{\mathrm{L}}=-\omega_{\mathrm{L}} \hat{\xi}_{\mathrm{L}}+u_{\mathrm{L}}, \\
& y_{\mathrm{L}}=\hat{\xi}_{\mathrm{L}},
\end{aligned}
$$

with $\omega_{\mathrm{L}}=3 \pi$. It approximates the double integration behaviour well above $8 \mathrm{~Hz}$. The additional lead-lag compensator $G_{11,1}$ given by

$$
\begin{aligned}
& \dot{\hat{\xi}}_{11, k}=-p_{k} \hat{\xi}_{11, k}+u_{11, k}, \\
& y_{11, k}=\left(z_{k}-p_{k}\right) \hat{\xi}_{11, k}+u_{11, k},
\end{aligned}
$$

with $p_{1}=0.6 \pi$ and $z_{1}=10 \pi$ is introduced to reduce the phase error in the low frequency regime around $8 \mathrm{~Hz}$ and bring it closer to the ideal $-180^{\circ}$. Due to discretization, the phase drops for higher frequencies, which can be compensated by a second lead-lag element $G_{11,2}$ with $p_{2}=103 \pi$ and $z_{2}=98 \pi$. The effect of $G_{112}$ on the overall transfer function degrades at higher sample rates and it can be omitted. The overall filter transfer function $G_{\mathrm{F}}$ is given by:

$$
\begin{aligned}
G_{\mathrm{F}}(s) & =G_{\mathrm{H}}(s) G_{\mathrm{L}}(s) G_{\mathrm{H}}(s) G_{\mathrm{L}}(s) G_{\mathrm{H}}(s) G_{\mathrm{ll}, 1}(s) G_{\mathrm{ll}, 2}(s) \\
& =G_{\mathrm{H}}^{3}(s) G_{\mathrm{L}}^{2}(s) G_{\mathrm{ll}, 1}(s) G_{\mathrm{ll}, 2}(s) .
\end{aligned}
$$

According to (3) with $E(s)=G_{\mathrm{F}}(s)$ it holds:

$$
X(s)=G_{\mathrm{F}}(s) Y(s) .
$$

which corresponds to the following state space representation:

$$
\begin{aligned}
\dot{\hat{\boldsymbol{\xi}}}(t) & =\boldsymbol{A} \hat{\boldsymbol{\xi}}(t)+\boldsymbol{B} y(t) \\
x(t) & =\boldsymbol{C} \hat{\boldsymbol{\xi}}(t) .
\end{aligned}
$$

with

$$
\boldsymbol{A}=\left(\begin{array}{ccccccc}
-p_{2} & 0 & 0 & 0 & 0 & 0 & 0 \\
z_{2}-p_{2} & -p_{1} & 0 & 0 & 0 & 0 & 0 \\
z_{2}-p_{2} & z_{1}-p_{1} & -\omega_{\mathrm{H}} & 0 & 0 & 0 & 0 \\
z_{2}-p_{2} & z_{1}-p_{1} & -\omega_{\mathrm{H}} & -\omega_{\mathrm{L}} & 0 & 0 & 0 \\
0 & 0 & 0 & 1 & -\omega_{\mathrm{H}} & 0 & 0 \\
0 & 0 & 0 & 1 & -\omega_{\mathrm{H}} & -\omega_{\mathrm{L}} & 0 \\
0 & 0 & 0 & 0 & 0 & 1 & -\omega_{\mathrm{H}}
\end{array}\right),
$$

$\boldsymbol{B}=\left(\begin{array}{lllllll}1 & 1 & 1 & 1 & 0 & 0 & 0\end{array}\right)^{\top}$,

$\boldsymbol{C}=\left(\begin{array}{lllllll}0 & 0 & 0 & 0 & 0 & 1 & -\omega_{\mathrm{H}}\end{array}\right)$

The bode diagram for $G_{\mathrm{F}}(s)$ is shown in Figure 5. While the double integrating behaviour is matched nicely in the regime of $8 \mathrm{~Hz}$ to $60 \mathrm{~Hz}$, there is a transition region between $0.8 \mathrm{~Hz}$ and $3 \mathrm{~Hz}$, within which signals are not sufficiently attenuated. Thus, this algorithm is ineffective if there is large system or measurement noise in this regime, as it might be the case for other accelerometers, depending on measurement principle and properties of the electronics. The estimator can be adjusted for different frequency ranges of desired double integration behaviour by changing $\omega_{\mathrm{H}}$, and $\omega_{\mathrm{L}}$ along with the lead lag poles $p_{k}$ and zeros $z_{k}$ appropriately.

For any estimator $G_{\mathrm{F}}(s)$, the theoretical minimal residual in case of ideal measurements can be calculated by evaluating the difference of the filter to the ideal, but not implementable double integrator. According to [15], for a normalized signal of amplitude 1 and frequency $\omega_{\mathrm{e}}$ the amplitude of this difference error $e(t)=\alpha_{\mathrm{e}} \sin \left(\omega_{\mathrm{e}} t-\beta_{\mathrm{e}}\right)$ is given by:

$$
\alpha_{\mathrm{e}}=\sqrt{1-2 M \cos (\Delta \varphi)+M^{2}},
$$

where $\Delta \varphi$ is the difference between the phase of $G_{\mathrm{F}}\left(j \omega_{\mathrm{e}}\right)$ and the ideal $-180^{\circ}$ and $M$ is the Amplitude quotient of $G_{\mathrm{F}}\left(j \omega_{\mathrm{e}}\right)$ and the ideal $\frac{1}{\omega_{\mathrm{e}}^{2}}$. For the given estimator $G_{\mathrm{F}}(s)$ in equation (10), this error is shown in Figure 6. As can be seen, the estimation error is mostly well below $5 \%$. However, as illustrated in Figure 7, the error grows fast for larger phase errors, which is the case for large delays. Even for a perfect amplitude estimation, the error can only remain below $5 \%$ for a phase error $<2.86^{\circ}$ and below $10 \%$ for a phase error 


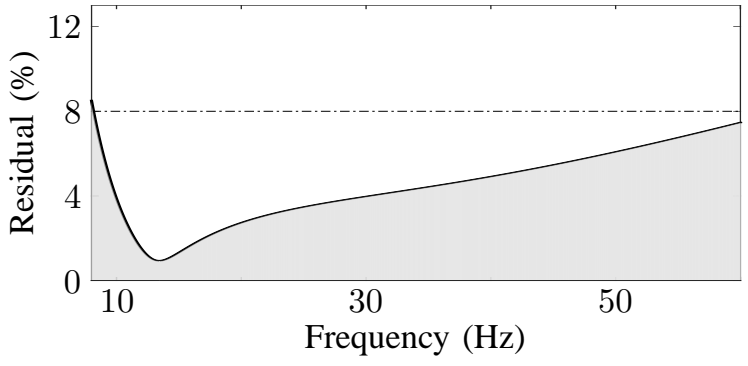

Fig. 6. Theoretical estimation error for the desired frequency range of $8 \mathrm{~Hz}$ to $60 \mathrm{~Hz}$ in case of undisturbed acceleration measurements.

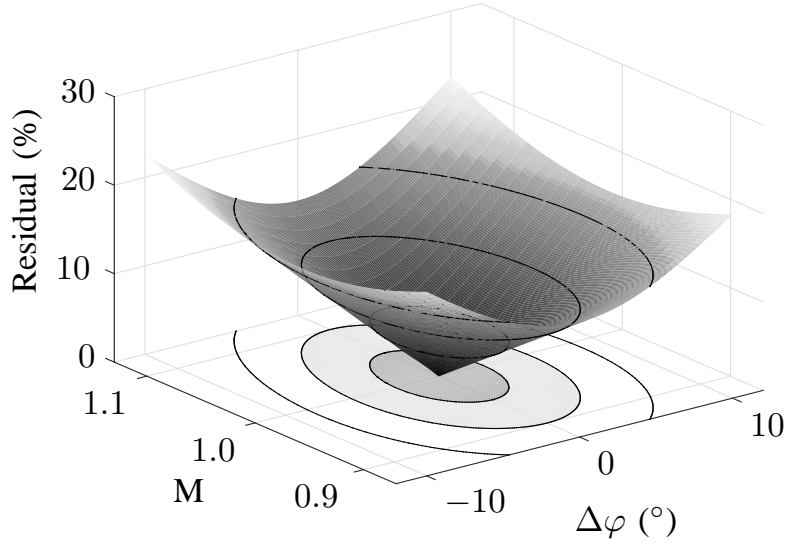

Fig. 7. 3D-plot of the error function from equation (13). The dark gray and the light gray elliptic region illustrate the area of about $5 \%$ and $10 \%$ estimation error, respectively.

$<5.73^{\circ}$. For the typical and very present disturbance frequency of $13 \mathrm{~Hz}$, which is well within our reference frequency range, this is equal to a delay of $1.14 \mathrm{~ms}$. Thus, even for small delays, a compensation can achieve noticable reduction of the residual RMS. In the next section, we will present a delay compensation algorithm which is tailored towards non-model based estimators such as $G_{\mathrm{F}}(s)$ derived in this section.

\section{Delay Compensation Algorithm}

\section{A. Derivation}

At the LBT, due to measurement distribution over a local area network using the UDP-Multicast protocol, the accelerometer signals are subject to a delay of approximately $T_{\mathrm{d}}=3 \mathrm{~ms}$. As typical for network delays, this value is not constant, instead it is distributed over a range from about $2.7 \mathrm{~ms}$ to $3.3 \mathrm{~ms}$. However, because the position is estimated before it is transmitted via UDP-Multicast, this delay jitter is not considered here, as it is not possible to predict the network error. Moreover, with this small jitter, there is no significant performance loss. In general, the concept explored in the following would be similar for a time-varying delay, with some modifications to be adopted from chapter 6.3 of [22]. According to (13), even with $\Delta \varphi=0$ and $M=1$ (perfect estimation for the undelayed case), this delay leads to a best case compensation ability of

$$
\alpha_{\mathrm{e}, \mathrm{opt}}=\sqrt{2-2 \cos \left(2 \pi f T_{\mathrm{d}}\right)}
$$

if the time delay is left uncompensated. For a $24 \mathrm{~Hz}$ sine disturbance, this best case residual error will remain at about $40 \%$ of the original value, for example. This illustrates the importance of a delay compensation algorithm.

There exist several traditional delay compensation algorithms, with the most famous being the smith predictor [23]. This is not applicable to our case, as it not only requires a plant model, but is furthermore a tool for overcoming dead time in closed loop control. In discrete control systems, the delay can be expressed in a linear fashion, simplifying the observer design. More general concepts developed by [22], [24] for many different kinds of sensor and/or actuator delays exist, but also rely on an actual model of the plant. Thus, for the broadband estimator designed in section II, these methods are not directly applicable, because our observer was not derived based on a plant model. Existing model-free delay compensation most often employs filtering with additional phase lead in order to compensate for the delay for specific frequencies. However, these methods require much tuning effort, which can be avoided with the algorithm presented in the following.

To compensate for the delay, the estimator from equation (12) has to be extended in order to increase the phase in the desired frequency regime. The method we choose to derive such an extended estimator is backstepping applied to a transport PDE representing the measurement delay and cascaded with a virtual system, which is extracted from the original disturbance estimator (12). This method is adapted from [22], but instead of an actual plant model, we extract a virtual model from our estimator. For this, let us rewrite the estimator given in (12) in a luenberger observer scheme [25]:

$$
\begin{aligned}
\dot{\hat{\boldsymbol{\xi}}}(t) & =\left(\boldsymbol{A}+\boldsymbol{B} \boldsymbol{C}_{z}\right) \hat{\boldsymbol{\xi}}(t)+\boldsymbol{B}\left(y(t)-\boldsymbol{C}_{z} \hat{\boldsymbol{\xi}}(t)\right), \\
\hat{z}(t) & =\boldsymbol{C}_{z} \hat{\boldsymbol{\xi}}(t), \\
x(t) & =\boldsymbol{C} \hat{\boldsymbol{\xi}}(t),
\end{aligned}
$$

with $z=\boldsymbol{C}_{z} \boldsymbol{\xi}$ being a fictitious output and

$$
\boldsymbol{C}_{z}=[-1,0,0,0,0,0,0] .
$$

This choice of $\boldsymbol{C}_{z}$ guarantees the stability of the observed autonomous system

$$
\begin{aligned}
\dot{\boldsymbol{\xi}}_{i}(t) & =\left(\boldsymbol{A}+\boldsymbol{B} \boldsymbol{C}_{z}\right) \boldsymbol{\xi}(t) \\
z(t) & =\boldsymbol{C}_{z} \boldsymbol{\xi}(t),
\end{aligned}
$$

due to the structure of $\boldsymbol{A}$ (lower triangular), since

$$
\begin{aligned}
& \boldsymbol{A}+\boldsymbol{B} \boldsymbol{C}_{z}= \\
& \left(\begin{array}{ccccccc}
-p_{2}-1 & 0 & 0 & 0 & 0 & 0 & 0 \\
z_{2}-p_{2}-1 & -p_{1} & 0 & 0 & 0 & 0 & 0 \\
z_{2}-p_{2}-1 & z_{1}-p_{1} & -\omega_{\mathrm{H}} & 0 & 0 & 0 & 0 \\
z_{2}-p_{2}-1 & z_{1}-p_{1} & -\omega_{\mathrm{H}} & -\omega_{\mathrm{L}} & 0 & 0 & 0 \\
0 & 0 & 0 & 1 & -\omega_{\mathrm{H}} & 0 & 0 \\
0 & 0 & 0 & 1 & -\omega_{\mathrm{H}} & -\omega_{\mathrm{L}} & 0 \\
0 & 0 & 0 & 0 & 0 & 1 & -\omega_{\mathrm{H}}
\end{array}\right)
\end{aligned}
$$

For the parameter values proposed in section II, it is possible to choose $\boldsymbol{C}_{z}=\boldsymbol{C}$. However, this might in general not yield a stable autonomous system (17). The transformation from (12) 
to (15) is illustrated in Figure 8a.

The measurement delay $T_{\mathrm{d}}$ is now appended to the output of the autonomous system (17) with the new delayed output $z_{\mathrm{d}}(t)$. The delay is modeled by a partial differential equation (PDE), commonly known as the transport equation.:

$$
\begin{aligned}
\dot{\boldsymbol{\xi}}(t) & =\left(\boldsymbol{A}+\boldsymbol{B} \boldsymbol{C}_{z}\right) \boldsymbol{\xi}(t) \\
u\left(T_{\mathrm{d}}, t\right) & =\boldsymbol{C}_{z} \boldsymbol{\xi}(t), \\
\partial_{t} u(v, t) & =\partial_{v} u(v, t), \\
z_{\mathrm{d}}(t) & =u(0, t) .
\end{aligned}
$$

with $u(v, t) \in\left[0, T_{\mathrm{d}}\right] \times[0, \infty)$ being a distributed state to model the infinite dimensional delay $T_{\mathrm{d}}$. The partial derivatives with respect to $t$ and $v$ are denoted by $\partial_{t}$ and $\partial_{v}$, respectively. Then, according to [24], the following observer guarantees asymptotically stable observer error dynamics using now the delayed measurement $y_{\mathrm{d}}(t)$ :

$$
\begin{aligned}
\dot{\hat{\boldsymbol{\xi}}}(t)= & \left(\boldsymbol{A}+\boldsymbol{B} \boldsymbol{C}_{z}\right) \hat{\boldsymbol{\xi}}(t) \\
& +\mathrm{e}^{\left(\boldsymbol{A}+\boldsymbol{B} \boldsymbol{C}_{z}\right) T_{\mathrm{d}}} \boldsymbol{B}\left(y_{\mathrm{d}}(t)-\hat{z}_{\mathrm{d}}(t)\right), \\
\partial_{t} \hat{u}(v, t)= & \partial_{v} \hat{u}(v, t) \\
& +\boldsymbol{C}_{z} \mathrm{e}^{\left(\boldsymbol{A}+\boldsymbol{B} \boldsymbol{C}_{z}\right) v} \boldsymbol{B}\left(y_{\mathrm{d}}(t)-\hat{z}_{\mathrm{d}}(t)\right), \\
\hat{u}\left(T_{\mathrm{d}}, t\right)= & \boldsymbol{C}_{z} \hat{\boldsymbol{\xi}}(t), \\
\hat{z}_{\mathrm{d}}(t)= & \hat{u}(0, t) .
\end{aligned}
$$

In order to get a predicted estimate of $x(t)$, which shall be called $x_{p}(t)$, the estimated state $\hat{\boldsymbol{\xi}}(t)$ can be used with:

$$
x_{\mathrm{p}}(t)=\boldsymbol{C} \hat{\boldsymbol{\xi}}(t) \text {. }
$$

A comparison of the delay compensating and the original observer is shown in Figure 8b. The additional Transport PDE can be seen on the right modeling the measurement delay. Because the PDE state has to be estimated as well, the extension is shown in the second box with the prediction $\mathrm{e}^{\left(\boldsymbol{A}+\boldsymbol{B} \boldsymbol{C}_{y}\right) T_{\mathrm{d}}}$ for the observer of the ordinary differential equation (ODE), and the distributed prediction $\mathrm{e}^{\left(\boldsymbol{A}+\boldsymbol{B} \boldsymbol{C}_{z}\right) v}$ for the PDE part of the observer.

\section{B. Dynamic properties}

To investigate the dynamic properties of the delay compensation, we can look at the transfer function of the delay compensating observer given by (19). This yields:

$$
\begin{aligned}
\hat{\boldsymbol{\Xi}}(s)= & \left(s \boldsymbol{I}-\boldsymbol{A}-\boldsymbol{B C}_{z}\right)^{-1} \\
& \cdot \mathrm{e}^{\left(\boldsymbol{A}+\boldsymbol{B} \boldsymbol{C}_{z}\right) T_{\mathrm{d}}} \boldsymbol{B}\left(Y_{\mathrm{d}}(s)-\hat{Z}_{\mathrm{d}}(s)\right), \\
\partial_{v} \hat{U}(v, s)= & s \hat{U}(v, s) \\
& -\boldsymbol{C}_{z} \mathrm{e}^{\left(\boldsymbol{A}+\boldsymbol{B} \boldsymbol{C}_{z}\right) v} \boldsymbol{B}\left(Y_{\mathrm{d}}(s)-\hat{Z}_{\mathrm{d}}(s)\right), \\
\hat{U}\left(T_{\mathrm{d}}, s\right)= & \boldsymbol{C}_{z} \hat{\boldsymbol{\Xi}}(s), \\
\hat{Z}_{\mathrm{d}}(s)= & \hat{U}(0, s), \\
X_{\mathrm{p}}(s)= & \boldsymbol{C} \hat{\boldsymbol{\Xi}}(s) .
\end{aligned}
$$

Using (22) and (24) we can solve for $\hat{U}(z, s)$ :

$$
\begin{array}{r}
\hat{U}(v, s)=\mathrm{e}^{s v}\left[\hat{U}(0, s)-\boldsymbol{C}_{z} \int_{0}^{v} \mathrm{e}^{-\left(s I-\boldsymbol{A}-\boldsymbol{B} \boldsymbol{C}_{z}\right) \zeta} \mathrm{d} \zeta\right. \\
\left.\cdot \boldsymbol{B}\left(Y_{\mathrm{d}}(s)-\hat{Z}_{\mathrm{d}}(s)\right)\right] \\
=\mathrm{e}^{s v} \hat{Z}_{\mathrm{d}}(s)+\boldsymbol{C}_{z}\left(s I-\boldsymbol{A}-\boldsymbol{B} \boldsymbol{C}_{z}\right)^{-1} \\
\cdot\left(\mathrm{e}^{\left(\boldsymbol{A}-\boldsymbol{B} \boldsymbol{C}_{z}\right) v}-\mathrm{e}^{s v}\right) \boldsymbol{B}\left(Y_{\mathrm{d}}(s)-\hat{Z}_{\mathrm{d}}(s)\right)
\end{array}
$$

Substituting (21) into (23) and using (26) with $v=T_{\mathrm{d}}$ yields:

$$
\begin{aligned}
\boldsymbol{C}_{z} \hat{\boldsymbol{\Xi}}(s)=\boldsymbol{C}_{z}\left(s I-\boldsymbol{A}-\boldsymbol{B C}_{z}\right)^{-1} & \cdot \mathrm{e}^{\left(\boldsymbol{A}+\boldsymbol{B} \boldsymbol{C}_{z}\right) T_{\mathrm{d}}} \boldsymbol{B}\left(Y_{\mathrm{d}}(s)-\hat{Z}_{\mathrm{d}}(s)\right) \\
=\mathrm{e}^{s T_{\mathrm{d}}} & \hat{Z}_{\mathrm{d}}(s)+\boldsymbol{C}_{z}\left(s I-\boldsymbol{A}-\boldsymbol{B} \boldsymbol{C}_{z}\right)^{-1} \\
& \cdot\left(\mathrm{e}^{\left(\boldsymbol{A}-\boldsymbol{B} \boldsymbol{C}_{z}\right) T_{\mathrm{d}}}-\mathrm{e}^{s T_{\mathrm{d}}}\right) \boldsymbol{B}\left(Y_{\mathrm{d}}(s)-\hat{Z}_{\mathrm{d}}(s)\right)
\end{aligned}
$$

Separating $\hat{Z}_{\mathrm{d}}(s)$ from $Y_{\mathrm{d}}(s)$, one can find:

$$
\begin{aligned}
\hat{Z}_{\mathrm{d}}(s)\left(\mathrm{e}^{s T_{\mathrm{d}}}\right. & \left.+\boldsymbol{C}_{z}\left(s I-\boldsymbol{A}-\boldsymbol{B} \boldsymbol{C}_{z}\right)^{-1} \boldsymbol{B} \mathrm{e}^{s T_{\mathrm{d}}}\right) \\
& =Y_{\mathrm{d}}(s) \boldsymbol{C}_{z}\left(s I-\boldsymbol{A}-\boldsymbol{B} \boldsymbol{C}_{z}\right)^{-1} \boldsymbol{B} \mathrm{e}^{s T_{\mathrm{d}}} \\
\Rightarrow \quad \hat{Z}_{\mathrm{d}}(s) & =\underbrace{\frac{\boldsymbol{C}_{z}\left(s I-\boldsymbol{A}-\boldsymbol{B} \boldsymbol{C}_{z}\right)^{-1} \boldsymbol{B}}{1+\boldsymbol{C}_{z}\left(s I-\boldsymbol{A}-\boldsymbol{B} \boldsymbol{C}_{z}\right)^{-1} \boldsymbol{B}}}_{=G_{\hat{z}, \mathrm{~d}}(s)} Y_{\mathrm{d}}(s) .
\end{aligned}
$$

Thus, the transfer function $G_{\hat{z}, \mathrm{~d}}(s)$ from $Y_{\mathrm{d}}(s)$ to $\hat{Z}_{\mathrm{d}}(s)$ is equal to the transfer function $G_{\hat{z}}(s)$ from $Y(s)$ to $Z(s)$ of the observer for the undelayed case, which can easily be calculated from (15). Now, for comparison, we will calculate the transfer function from the measurement $Y_{\mathrm{d}}(s)$ to the predicted estimate $X_{p}(s)$. Substituting (29) into (21) now yields:

$$
\hat{\boldsymbol{\Xi}}(s)=\frac{\left(s I-\boldsymbol{A}-\boldsymbol{B} \boldsymbol{C}_{z}\right)^{-1} \mathrm{e}^{\left(\boldsymbol{A}+\boldsymbol{B} \boldsymbol{C}_{z}\right) T_{\mathrm{d}}} \boldsymbol{B}}{1+\boldsymbol{C}_{z}\left(s I-\boldsymbol{A}-\boldsymbol{B} \boldsymbol{C}_{z}\right)^{-1} \boldsymbol{B}} Y_{\mathrm{d}}(s),
$$

and thus with (25)

$$
\begin{aligned}
X_{\mathrm{p}}(s) & =\boldsymbol{C} \hat{\boldsymbol{\Xi}}(s) \\
= & \underbrace{\frac{\boldsymbol{C}\left(s I-\boldsymbol{A}-\boldsymbol{B} \boldsymbol{C}_{z}\right)^{-1} \mathrm{e}^{\left(\boldsymbol{A}+\boldsymbol{B} \boldsymbol{C}_{z}\right) T_{\mathrm{d}} \boldsymbol{B}}}{1+\boldsymbol{C}_{z}\left(s I-\boldsymbol{A}-\boldsymbol{B} \boldsymbol{C}_{z}\right)^{-1} \boldsymbol{B}}}_{G_{x, \mathrm{~d}}(s)} Y_{\mathrm{d}}(s) .
\end{aligned}
$$

The transfer function from the undelayed measurement $Y(s)$ to the estimated output $X(s), G_{x}(s)$, can be calculated from system (15):

$$
X(s)=\underbrace{\frac{\boldsymbol{C}\left(s I-\boldsymbol{A}-\boldsymbol{B} \boldsymbol{C}_{z}\right)^{-1} \boldsymbol{B}}{1+\boldsymbol{C}_{z}\left(s I-\boldsymbol{A}-\boldsymbol{B} \boldsymbol{C}_{z}\right)^{-1} \boldsymbol{B}}}_{G_{x}(s)} Y(s) .
$$

The transfer function $G_{x, \mathrm{~d}}$ from (31) is identical to the transfer function $G_{x}(s)$ from (32) , except for the additional matrix $\mathrm{e}^{\left(\boldsymbol{A}+\boldsymbol{B} \boldsymbol{C}_{y}\right) T_{\mathrm{d}}}$, which introduces the necessary phase lead in order to compensate for the delay. For comparison, the bode diagram of the delay compensating estimator $G_{x, \mathrm{~d}}$, the estimator $G_{x, \mathrm{~d}}$ in series with the delay function $\mathrm{e}^{-s T_{\mathrm{d}}}$, and that of the ideal double integrator are displayed in Figure 9. 

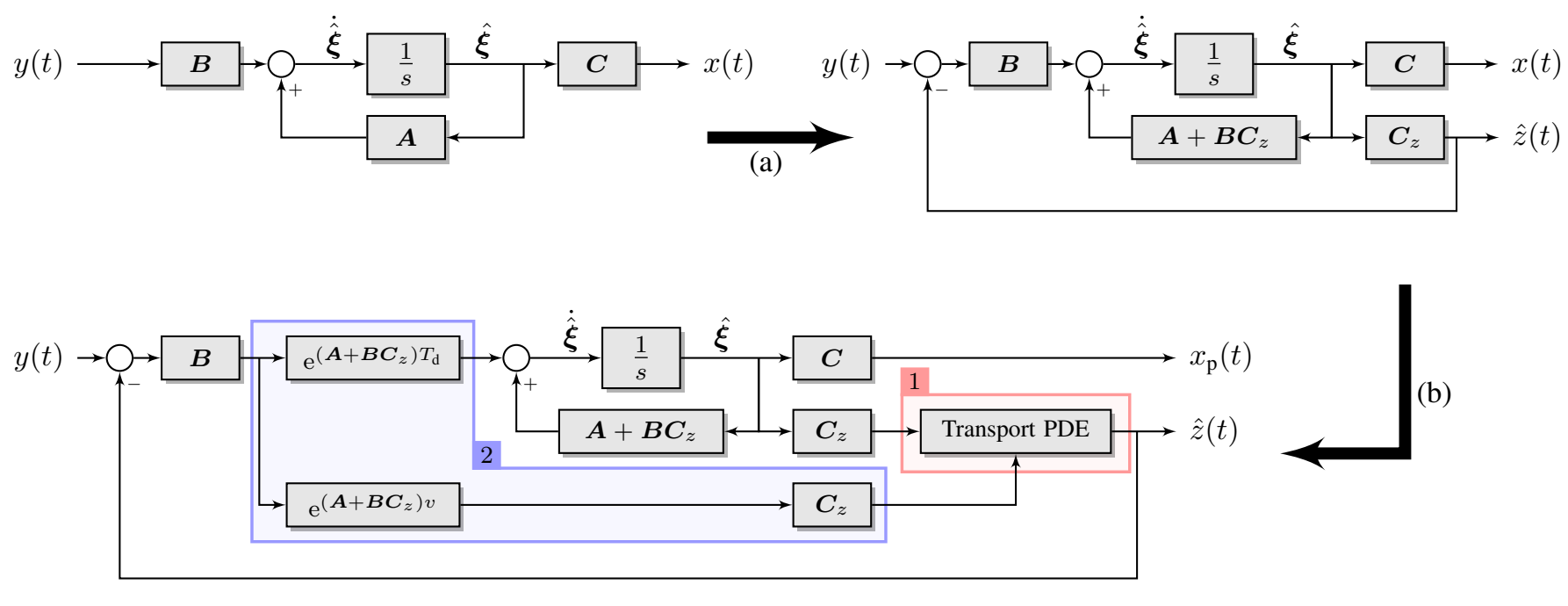

Fig. 8. (a) Transformation of the observer scheme (12) into (15) and (b) extension to the full delay compensating observer scheme (19) featuring additional measurement delay ( 1 - red box) and the delay compensating extensions to the original observer feedback ( 2 - blue box).

The phase lead of the compensating estimator can be seen clearly for higher frequencies.

Classical methods for delay compensation rely on a good plant model in order to extrapolate the state successfully into the future, or require a lot of tuning effort, like non model based strategies which employ additional phase lead elements to compensate the delay. The proposed delay compensation algorithm however does not rely on a model, nor does it need any additional tuning parameters. On the downside, the algorithm's compensating ability drops significantly for $T_{\mathrm{d}}>15 \mathrm{~ms}$, for which model-based strategies can be much more successful, if a very realistic model is employed and all the inputs and disturbances are accurately known in advance. In general, the prediction performance is always tied to the fidelity of the model. Since the described algorithm is based on a virtual model with no physical reference, the prediction performance cannot surpass such of model based algorithms.

\section{Implementation}

While the design and analysis of the filter and the delay compensation was performed in the previous sections in the frequency domain, the implementation is based on an exact discretized state space version of (19). As can be seen in equation (31), even though the observer contains an infinite dimensional PDE, the transfer function from $Y(s)$ to $X_{\mathrm{p}}(s)$ has a finite number of poles and zeros. Hence, an exact discretization based on sample and hold measurements is possible. The sampling frequency is $1 \mathrm{kHz}$ in the simulation and for the implementation at the LBT. The small phase lag relevant for higher frequencies due to the sampling is compensated for already in the original observer with an appropriate lead-lag element, $G_{11,2}(s)$ as introduced in equation (10). Thus the performance will not be affected in the relevant frequency regime of $5 \mathrm{~Hz}$ to $60 \mathrm{~Hz}$ by using a discretized version of (19). The following results are based on simulations of the discretized algorithm using actual measurements as the input.
At the LBT, the algorithm is currently being implemented on a PowerPC with a $1 \mathrm{GHz}$ processor and $1 \mathrm{~GB}$ of memory, which seems more than enough power to handle this algorithm with $1 \mathrm{kHz}$ sampling.

\section{Measurement Results From LBT}

Two interferometers are foreseen to be installed at the LBT in its final state. One of them is the LBT Interferometer (LBTI), which in contrast to LINC-NIRVANA is already installed at the LBT and is currently undergoing commissioning until it reaches its full operational capabilities. For this, among many other things, it is very important to compensate the OPD induced by telescope mirror vibrations in order to see and stabilize the desired fringe pattern on the detector. Thanks to the LBTI group it was possible to acquire open loop fringes of a very bright star together with the respective accelerometer data and apply our estimation scheme, in order to see whether or not a sufficient disturbance rejection can be reached, even though this analysis of course assumes an ideal, or at least very fast correction device. For LINC-NIRVANA, the correction device is the piston mirror, which has a two degree of freedom control architecture featuring a bandwith of about $100 \mathrm{~Hz}$. Due to the control design, the desired input is first smoothed using an FIR-filter introducing an additional delay of $\approx 5 \mathrm{~ms}$. Assuming linear system behaviour for the telescope and the piston mirror, this delay can simply be added to the communication delay of the measurement values. Due to LBTI's tweeter-woofer approach to the correction, control is much simpler for it's correction devices and there is no additional input delay.

Refer again to Figure 2 from the beginning for the optical setup at the LBT and LBTI. The LBTI fringe tracking module for this experiment was the PHASECam, the instrument's two correction devices to correct for slow, but large (SPC), and fast, but small (FPC) amplitude oscillations in the OPD were unused, i. e. turned off and fixed for this experiment - LBTI was run in so called open-loop mode. This way, fringes tracked 

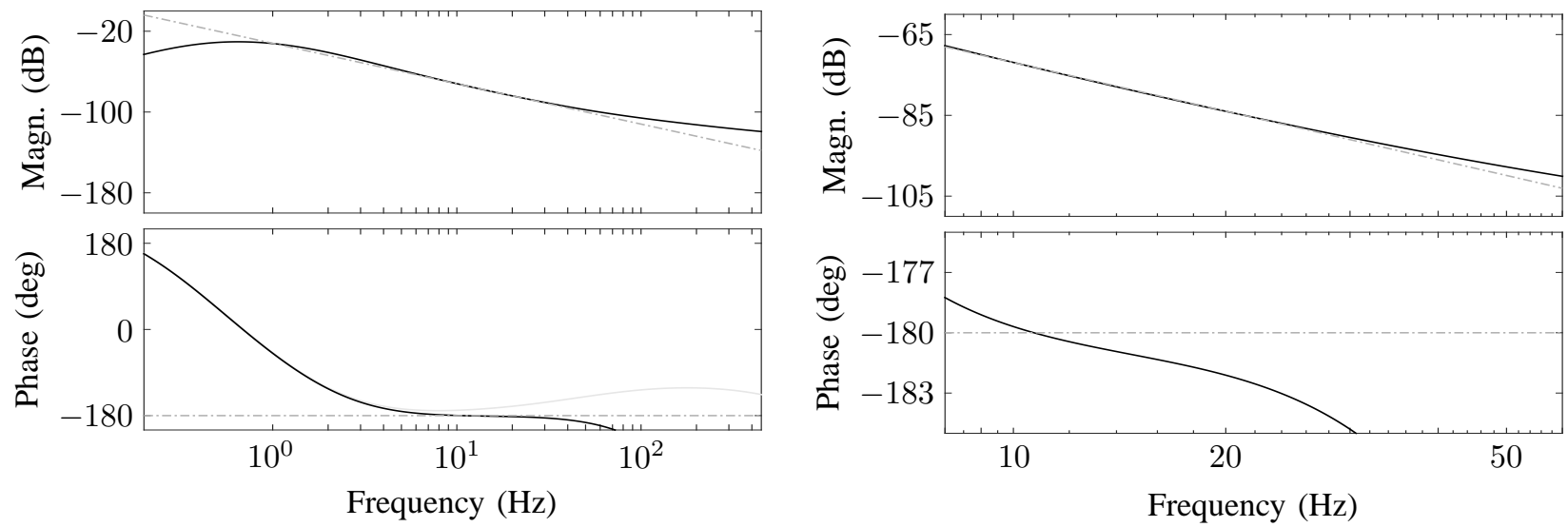

Fig. 9. Bode Diagramm comparing the ideal double integrator (dash-dotted gray) with the designed filter with delay compensation in series with the respective delay, $G_{x, \mathrm{~d}}(s) \mathrm{e}^{-s T_{\mathrm{d}}}$ (solid dark). On the left, the bode diagram for the complete frequency range is shown, on the right a zoom in for the desired frequency range of $8 \mathrm{~Hz}$ to $60 \mathrm{~Hz}$ is shown. Additionaly, the phase of the estimator without the plant delay is shown on the left for comparison (light gray).

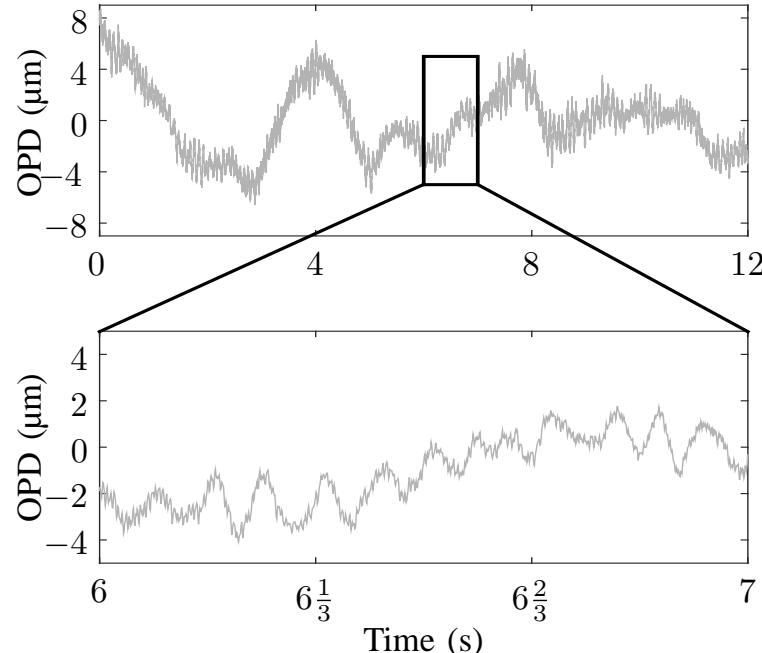

Fig. 10. OPD calculated by LBTI from tracked fringes at Feb. 4, 2015, 08:34:47 UTC.

by LBTI's PHASECam can be used to determine the total OPD between the left and right side of the telescope. An example time series taken on February 4, 2015 and starting at 08:34:47 UTC, is shown in Figure 10. In parallel, we recorded synchronized acclerometer data from all accelerometers. As can be seen, there are very slow oscillations $<2 \mathrm{~Hz}$, which are mostly due to atmospheric effects and the slow telescope tracking motion, while oscillations $>10 \mathrm{~Hz}$ are due to telescope vibrations. What looks like measurement noise is actually a $50 \mathrm{~Hz}$ oscillation with an amplitude of $<1 \mu \mathrm{m}$ and is due to internal vibrations of the instrument, which cannot be measured by the OVMS and therefore cannot be corrected. Additional accelerometers within the instrument along with the algorithm presented in [26], which is very well suited for single frequency disturbances, could in principle eliminate these disturbances very well, but because the actuator might excite the optical bench and its smaller optical parts, the controller design needs to be carried out carefully, to account for the physical feedback between the actuator and the vibrating optical components.

As can be seen in the timeseries in Figure 11, within the design frequency range of $8 \mathrm{~Hz}$ to $60 \mathrm{~Hz}$, the OPD RMS of $603 \mathrm{~nm}$ could be reduced by $61 \%$ to $234 \mathrm{~nm}$ without any compensation of the measurement delay. Using the proposed prediction algorithm to compensate the measurement delay of $T_{\mathrm{d}}=3 \mathrm{~ms}$, this can be further improved down to $192 \mathrm{~nm}$ (a rejection of around $68 \%$ of the disturbance), thus demonstrating the effectiveness of the proposed algorithm. All of these numbers are calculated under the assumption of an ideal correction with very fast correction devices, which is a reasonable assumption for both LBTI and LINC-NIRVANA.

By shifting the data in time for an offline analysis, we can compare the estimation results of the originally proposed estimator (12) and the estimator extended for delay compensation via backstepping (19) presented in section III. We will evaluate now the delay compensation for a delay of $8 \mathrm{~ms}$. This scenario is very important for LINC-NIRVANA, because its correction device is internally controlled by a two degreeof-freedom structure, and the feedforward is based on an inversion of dynamics of the compensation device. Thus, in order to generate a feasible feedforward control signal, high frequencies in the desired input need to be suppressed, for which a finite-impulse-response (FIR) filter is used. FIR-filter have a linear phase, i.e. the phase response can be modeled as a pure delay. However, the drawback of this is an additional input delay of $5 \mathrm{~ms}$. Thus, LINC-NIRVANA depends on a reliable prediction to compensate a total delay of $8 \mathrm{~ms}$. In general, as illustrated by Figure 12, the amplitude spectrum of the estimated and the measured OPD (by LBTI) match in a wide frequency range. $\mathrm{M} 3$ resonances $(13.5 \mathrm{~Hz}$ to $19 \mathrm{~Hz})$ are not matched as perfectly as $\mathrm{M} 2$ resonances $(10 \mathrm{~Hz}$ to $13 \mathrm{~Hz})$ due to a sensor fault at $\mathrm{M} 3$ on the right side of the telescope, which could not be fixed in time for the measurements. However, without the delay compensation, the disturbance is not rejected as desired and significant peaks in the residual amplitudes can be seen in the right of Figure 12, as predicted in the beginning of Section III. Utilizing the proposed delay compensating observer (19), all major resonances could be 

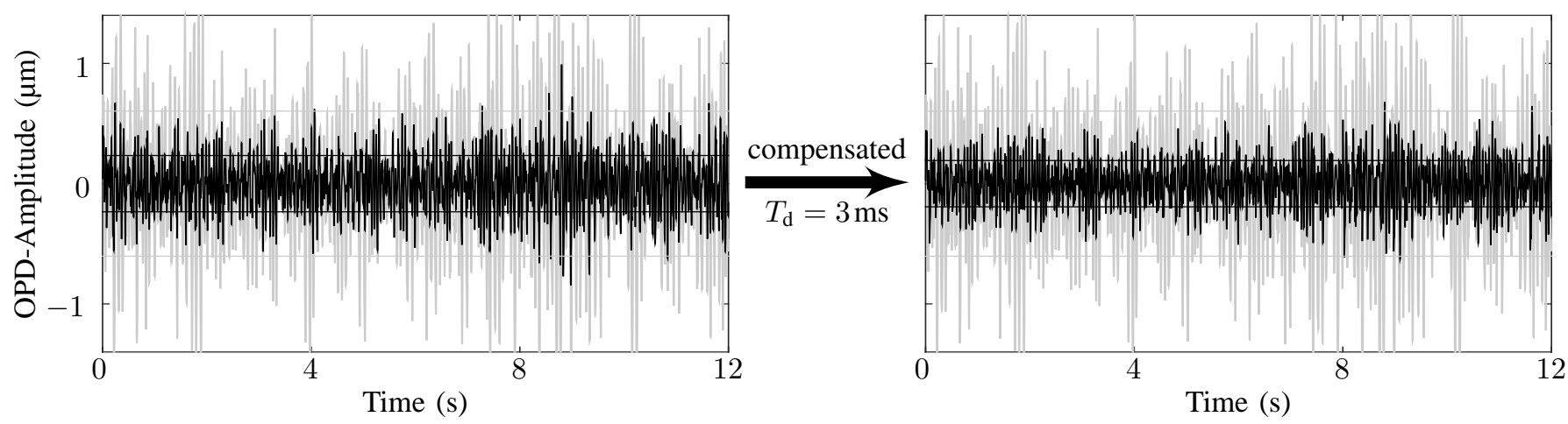

Fig. 11. On the left: time series of LBTI measured OPD (light) and OPD after correction using the OPD estimated with the orginial observer (12) (dark). On the right: time series of LBTI measured OPD (light) and OPD after correction using the OPD estimated with the delay compensating observer (19) (dark) to compensate for $3 \mathrm{~ms}$ of measurement delay. The straight lines illustrate the RMS-value of the residual signal (dark). The time series have been filtered using a type I Chebyshev filter of order 5 with a passband of $8 \mathrm{~Hz}$ to $60 \mathrm{~Hz}$.
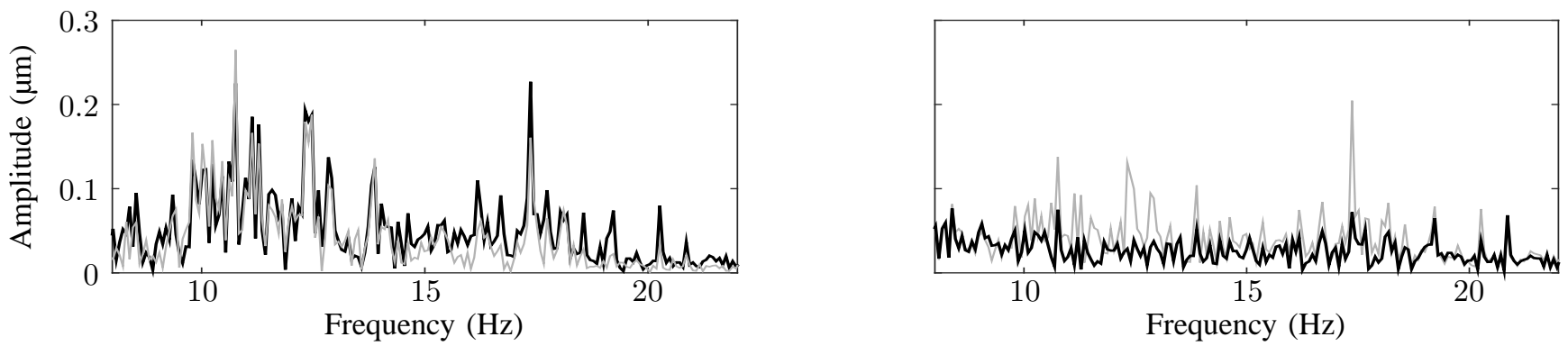

Fig. 12. On the left: amplitude spectrum of LBTI OPD measurement (dark) vs. OPD estimated from synchronized OVMS measurements (light). On the right: amplitude spectrum of the residual signal with (dark) and without (light) delay compensation for a delay of $8 \mathrm{~ms}$.

attenuated.

\section{CONCLUSION}

In this paper a method to compensate small measurement delays for model free filters has been presented and experimentally validated. While the application of the presented method is not limited to astronomy, the estimation of the OPD at the LBT is particularly challenging, because phase and amplitude have to be estimated very precisely, while the spectrum is very broad at the same time. However, the feasibility of the proposed estimator and the proposed delay compensation could be proven. It has been shown that delay compensation is necessary even for small delays for such a phase-sensitive application and that nearly the same performance in estimation can be achieved using the delayed measurements as compared to using undelayed measurements. Disturbances in the frequency regime of $8 \mathrm{~Hz}$ to $60 \mathrm{~Hz}$ could be attenuated by a factor $>3$ to less than $32 \%$ of the original level. Most of the remaining OPD is due to internal instrument vibrations. Some vibrations of the tertiary mirrors could not be compensated due to an unresolved sensor issue. With all sensors working, a better performance is expected, but there will remain presumably about $20 \%$ residual OPD originating from atmospheric aberrations and vibrations of the instrument optics. This part will never be compensated for in a disturbance feedforward utilizing only the main telescope mirrors. Thus, similar to the way this was done in [8] for the VLTI, a combination of feedforward and feedback techniques as well as mechanical design optimization will have to be used to further decrease the RMS of the remaining OPD to reach the goal of $0.1 \lambda$ RMS for smaller wavelength observed by LINCNIRVANA.

An additional advantage of the described accelerometer based system also lies in the fact that this system can be easily upgraded to measure more optics, for example.

Last, but not least, this method can help enhance any filtering performed on delayed measurements, where the delay is a limiting factor in the control loop's performance. This problem is becoming more severe with the rapid increase in the number of sensor in many application, which is often dealt with using bus systems such as ethernet, CAN-bus or others, resulting in significant delays of the sensor values.

\section{ACKNOWLEDGMENT}

The authors would like to thank the entire LBTI team and in particular Denis Defrère, whose continuous effort has made it possible to provide the relevant measurements used in this paper to evaluate the performance of the presented estimator.

\section{REFERENCES}

[1] L. Cavaller, J. Marrero, J. Castro, E. Morante, M. Ronquillo, and E. Hernndez, "Design of the primary mirror segment support system for the E-ELT," in Proc. SPIE, vol. 7012, 2008, pp. $70121 \mathrm{~F}-70121 \mathrm{~F}-11$. [Online]. Available: http://dx.doi.org/10.1117/12.789784 
[2] C. Baffes, T. Mast, J. Nelson, E. Ponslet, V. Stephens, L. Stepp, and E. C. Williams, "Primary mirror segmentation studies for the Thirty Meter Telescope," in Proc. SPIE, vol. 7018, 2008, pp. 70180 S70 180S-15. [Online]. Available: http://dx.doi.org/10.1117/12.790206

[3] M. Johns, C. Hull, G. Muller, B. Irarrazaval, A. Bouchez, T. Chylek, C. Smith, A. Wadhavkar, B. Bigelow, S. Gunnels, B. McLeod, and C. Buleri, "Design of the Giant Magellan Telescope," in Proc. SPIE, vol. 9145, 2014, pp. $91451 \mathrm{~F}-91451 \mathrm{~F}-14$. [Online]. Available: http://dx.doi.org/10.1117/12.2057286

[4] D. Defrére, P. Hinz, E. Downey, D. Ashby, V. Bailey, G. Brusa, J. Christou, W. C. Danchi, P. Grenz, J. M. Hill, W. F. Hoffmann, J. Leisenring, J. Lozi, T. McMahon, B. Mennesson, R. MillanGabet, M. Montoya, K. Powell, A. Skemer, V. Vaitheeswaran, A. Vaz, and C. Veillet, "Co-phasing the Large Binocular Telescope: status and performance of LBTI/PHASECam," in Proc. SPIE, vol. 9146, 2014, pp. 914609-914609-8. [Online]. Available: http://dx.doi.org/10.1117/12.2057178

[5] T. M. Herbst, R. Ragazzoni, A. Eckart, and G. Weigelt, "LINCNIRVANA: achieving 10 mas imagery on the Large Binocular Telescope," in Society of Photo-Optical Instrumentation Engineers (SPIE) Conference Series, ser. Society of Photo-Optical Instrumentation Engineers (SPIE) Conference Series, vol. 7014, Aug. 2008.

[6] M. Born and E. Wolf, Principles of Optics, 7th ed. Cambridge University Press, 2002.

[7] D. G. MacMynowski and T. Andersen, "Wind buffeting of large telescopes," Applied Optics, vol. 49, pp. 625-636, 2010.

[8] H. Bonnet, B. Bauvir, A. Wallander, M. Cantzler, J. Carstens, F. Caruso, N. di Lieto, S. Guisard, P. Haguenauer, N. Housen, M. Mornhinweg, J.L. Nicoud, A. Ramirez, J. Sahlmann, G. Vasisht, S. Wehner, and J. Zagal, "Enabling Fringe Tracking at the VLTI," The Messenger (published by ESO), vol. 126, pp. 37-40, Dec. 2006.

[9] J.-U. Pott, M. Kürster, M. Böhm, T. Ruppel, S. Engelke, J. Trowitzsch, J. Borelli, W. Gässler, R.-R. Rohloff, and T. Herbst, "Vibration control of ELTs," in Proc. of AO4ELT 2, 2012.

[10] M. Kürster, T. Bertram, J. L. Borelli, M. Brix, W. Gässler, T. M. Herbst, V. Naranjo, J.-U. Pott, and J. Trowitzsch, "OVMS: the optical path difference and vibration monitoring system for the LBT and its interferometers," in Proc. of SPIE, vol. 7734, Jul. 2010.

[11] A. Keck, J. Pott, and O. Sawodny, "An accelerometer based dual loop approach to minimize the impact of fast telescope vibrations seen by the E-ELT/MICADO wavefront sensors," in Adaptive Optics for Extremely Large Telescopes Conference 3, 2013.

[12] M. Brix, J.-U. Pott, T. Bertram, S. Rost, J. L. Borelli, T. M. Herbst, M. Kuerster, and R.-R. Rohloff, "Linc-Nirvana piston control elements," in Proc. SPIE, vol. 7734, Jul. 2010.

[13] R. N. Paschall and D. J. Anderson, "Linear quadratic gaussian control of a deformable mirror adaptive optics system with time-delayed measurements," Appl. Opt., vol. 32, no. 31, pp. 6347-6358, Nov 1993. [Online]. Available: http://ao.osa.org/abstract.cfm?URI=ao-32-31-6347

[14] L. Poyneer and J.-P. Véran, "Predictive wavefront control for adaptive optics with arbitrary control loop delays," J. Opt. Soc. Am. A, vol. 25 , no. 7, pp. 1486-1496, Jul 2008. [Online]. Available: http://josaa.osa.org/abstract.cfm?URI=josaa-25-7-1486

[15] M. Böhm, J.-U. Pott, O. Sawodny, T. Herbst, and M. Kürster, "RealTime Vibration Compensation for Large Telescopes," Monthly Notices of the Royal Astronomical Society, vol. 442, no. 3, pp. 2446-2455, 2014.

[16] M. Böhm, J.-U. Pott, M. Kürster, and O. Sawodny, "Modeling and Identification of the Optical Path at ELTs - a Case Study at the LBT," in Proc. of the 6th IFAC Symposium on Mechatronic Systems, vol. 6, April 2013, pp. 249-255.

[17] M. Bodson and S. C. Douglas, "Adaptive Algorithms for the Rejection of Sinusoidal Disturbances with Unknown Frequency," Automatica, vol. 33, pp. 2213-2221, 1997.

[18] A. De Luca, D. Schroder, and M. Thummel, "An Acceleration-based State Observer for Robot Manipulators with Elastic Joints," in IEEE International Conference on Robotics and Automation, April 2007, pp. $3817-3823$

[19] S. Engelke, L. Gaul, J.-U. Pott, M. Kürster, J. Trowitzsch, and J. L. Borelli, "Process Noise Identification and Observer Design for the Large Binocular Telescope," in Proceedings of the 30th IMAC, A Conference on Structural Dynamics, vol. 30, 2012, pp. 407-414.

[20] N. Di Lieto, J. Sahlmann, A. Wallander., and G. Vasisht, "An Approach to Stabilizing Large Telescopes for Stellar Interferometry," in Proc. of ICALEPCS, 2007

[21] H. Gilbert, O. Celik, and M. O'Malley, "Long-term double integration of acceleration for position sensing and frequency domain system identification," in IEEE/ASME International Conference on Advanced Intelligent Mechatronics (AIM), July 2010, pp. $453-458$.

[22] M. Krstic, Delay Compensation for Nonlinear, Adaptive, and PDE Systems, T. Başar, Ed. Birkhäuser, 2009.

[23] Smith, "A Controller to Overcome Dead-Time," Indian Scientist Association Journal, vol. 2, pp. 28-33, 1959.

[24] M. Krstic and A. Smyshlyaev, "Backstepping boundary control for firstorder hyperbolic PDEs and application to systems with actuator and sensor delays," Systems \& Control Letters, vol. 57, no. 9, pp. $750-$ $758,2008$.

[25] D. Luenberger, "Observing the State of a Linear System," Military Electronics, IEEE Transactions on, vol. 8, no. 2, pp. 74-80, April 1964

[26] A. Keck, J.-U. Pott, and O. Sawodny, "Accelerometer-based online reconstruction of vibrations in extremely large telescopes," in in Proc. IFAC World Congress, 2014, cape Town, 2014, pp. 7467-7473.

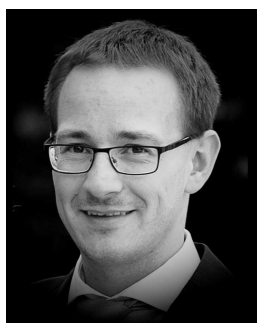

Michael Böhm received the Dipl.Ing. degree in engineering cybernetics from the University of Stuttgart, Stuttgart, Germany, in 2011.

$\mathrm{He}$ worked on control of coupled wave equations as a DAAD scholar at the Cymer Center for Control Systems and Dynamics at the University of California, San Diego from 2010 to 2011. Since 2011, he has been holding a Ph.D. research position with the institute for System Dynamics, University of Stuttgart, Stuttgart, Germany. His main research interests include dynamic filtering, disturbance compensation, and delay compensation as well as modeling and control of distributed parameter systems in general and adaptive optics systems in particular

Jörg-Uwe Pott ...

Martin Kürster ...

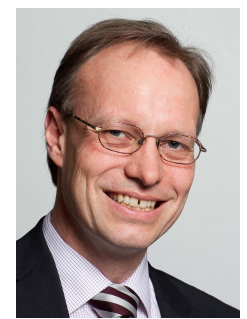

Oliver Sawodny received the Dipl.Ing. degree in electrical engineering from the University of Karlsruhe, Karlsruhe, Germany, in 1991, and the Ph.D. degree from the University of Ulm, Ulm, Germany, in 1996.

In 2002, he became a Full Professor with the Technical University of Ilmenau, Ilmenau, Germany. Since 2005, he has been the Director of the Institute for System Dynamics, University of Stuttgart, Stuttgart, Germany. His current research interests include methods of differential geometry, rajectory generation, and applications to mechatronic systems.

Denis Defrère ...

Phil Hinz . . 